\title{
The ambulatory arterial stiffness index and target-organ damage in Chinese patients with chronic kidney disease
}

\author{
Cheng Wang ${ }^{\dagger}$, Jun Zhang ${ }^{\dagger}$, Cui-Cui Li, Wen-Yu Gong, Xun Liu, Zeng-Chun Ye, Hui Peng and Tan-Qi Lou*
}

\begin{abstract}
Background: The ambulatory arterial stiffness index (AASI) can be used to predict cardiovascular morbidity and mortality in hypertensive patients. However, data on AASI in Chinese patients with chronic kidney disease (CKD) is not available.

Methods: This cross-sectional study enrolled 583 CKD patients. Univariate and multivariate analyses were used to evaluate the relationship between AASI and renal function and parameters of cardiovascular injury.

Results: Patients with a higher AASI had a higher systolic blood pressure, a lower estimated glomerular filtration rate (eGFR), a higher serum cystatin C, a higher left ventricular mass index (LVMI) and carotid intima-media thickness (cIMT). Univariate analyses showed that AASI was positively correlated with serum cystatin $C(r=0.296, P<0.001)$, serum creatinine $(r=0.182, P<0.001)$, and LVMI $(r=0.205, P<0.001)$ and negatively correlated with the eGFR $(r=-0.200, P<0.001)$. Multivariate analyses revealed that serum cystatin $C$, eGFR, serum creatinine and LVMI were independently correlated with AASI.
\end{abstract}

Conclusions: These data suggest that AASI was closely correlated with renal function and parameters of cardiovascular injury in Chinese CKD patients. Good quality, long-term, large longitudinal trials to validate the role of AASI in clinical practice for Chinese CKD patients.

Keywords: Ambulatory arterial stiffness index, Chronic kidney disease, Renal function, Left ventricular mass index

\section{Background}

The rising prevalence and associated morbidity of chronic kidney disease (CKD) has resulted in a significant disease burden and became a major public health problem for many countries [1]. Cardiovascular disease (CVD) is the leading cause of premature death in patients with CKD [2], so research on vascular changes in CKD patients is very important. Reduced arterial elasticity has been observed in CKD patients [3]. Fibroelastic intimal thickening, an increased extracellular matrix, enhanced collagen density, and vascular calcification seem to contribute to "stiffer" arteries [4]. It has been demonstrated that vascular stiffness can predict adverse cardiovascular outcomes in patients with primary hypertension [5].

\footnotetext{
* Correspondence: lou.tq@163.com

${ }^{\dagger}$ Equal contributors

Division of Nephrology, Department of medicine, Third Affiliated Hospital of Sun Yat-Sen University, Guangzhou, Guangdong 510630, China
}

In 1914, MacWilliam and Melvin stated that a loss of elasticity in the arterial system influences diastolic blood pressure (DBP) and its relationship with systolic blood pressure (SBP). According to this basic principle, Li et al. proposed a novel, easy-to-obtain index of arterial stiffness: the ambulatory arterial stiffness index (AASI). AASI is defined as 1 minus the regression slope of DBP plotted against SBP obtained from individual 24-h blood pressure (BP) recordings. They also described the close correlation of AASI with pulse wave velocity (PWV) as well as central and peripheral augmentation indices [6]. Moreover, Dolan et al. showed that AASI can provide prognostic information because it was a predictor of stroke and cardiac death in a cohort of 11,291 patients [7]. Kikuya et al. also observed that the AASI predicted mortality due to CVD and stroke over and beyond pulse pressure [8]. Muxfeldt et al. demonstrated that AASI was a predictor of cardiovascular morbidity and mortality in 547 patients with resistant hypertension [9]. 
Furthermore, some research teams have reported that AASI has good reproducibility, with repeatability coefficients of $\leq 60 \%$ [10]. AASI has good correlation with target-organ damage in patients with primary hypertension $[11,12]$. However, some have criticized the significance of AASI in assessing arterial compliance, especially in pediatric populations [13,14]; what is more, few studies have focused on AASI in Chinese CKD patients. Investigating the relationship between AASI and target organ damage in Chinese CKD patients is very important when considering different life style, genetic factors, environment and the primary cause of CKD in the Chinese population. Hence, we carried out an observational study to identify the relationship between AASI and target organ damage in CKD patients residing in China.

\section{Methods}

\section{Design and population of the study}

The study protocol was approved by the ethics committee of $3{ }^{\text {rd }}$ Hospital of Sun Yat-sen University. All of the study participants provided written informed consent to be included in the study. Six hundred and fifty consecutive in patients with CKD were included from May 2010 to July 2012. A cross-sectional study was carried out in our division. The exclusion criteria were: treatment with corticosteroids or hormones; acute changes in the estimated glomerular filtration rate (eGFR) $>30 \%$ in the previous 3 months; pregnancy; history of abuse of drugs or alcohol; night or shift-work employment; acquired immunodeficiency syndrome; cardiovascular disorders (unstable angina pectoris, heart failure, life-threatening arrhythmia, atrial fibrillation and grade III-IV retinopathy); intolerance to ambulatory blood pressure monitoring (ABPM); inability to communicate and comply with all of the study requirements; or maintenance dialysis. We excluded 67 patients for abnormal ABPM or incomplete measurement of baseline characteristics. Therefore, 583 patients were enrolled into the study. Forty-nine subjects had diabetic nephropathy and 109 patients had immunoglobulin (Ig) A nephropathy in this group.

\section{Assessment}

1. ABPM: Patients underwent 24-h ABPM using a TM-2430 monitor (A\&D, Tokyo, Japan). Cuff size was chosen based on arm circumference. The cuff was fixed to the non-dominant arm. Three BP readings were obtained in the morning (07:00 to 10:00) concomitant with sphygmomanometric measurements to ensure that the average of the two sets of values differed by $\leq 5 \mathrm{mmHg}$. BP was recorded every $15 \mathrm{~min}$ from 07:00 to 22:00, and from every $30 \mathrm{~min}$ from 22:00 to 07:00. The values for daytime and nighttime periods were derived from diaries recorded by the patients during ABPM. Monitoring was done on a working day. Patients had no access to ABP values. Strenuous physical activity was discouraged in all patients during the monitoring period, and their daily activities were comparable.

BP series were eliminated from the analysis if: $>30 \%$ of the measurements were lacking; they had missing data for $>3$-h spans; they were collected from subjects who were experiencing an irregular rest-activity schedule or a nighttime sleep span $<6$ h or $>12 \mathrm{~h}$ during monitoring. Raw data were examined by scatter plots. The regression slope of DBP on SBP was computed to obtain the AASI (1-slope). The diagnosis of hypertension was based on accepted criteria for ABPM [15]: ambulatory blood pressure was considered to be "normal" if the mean awake BP was $<135 / 85 \mathrm{mmHg}$ for SBP/DBP or if the mean bedtime BP was $<120 / 70 \mathrm{mmHg}$.

2. BP measurement in the physician's office: BP was measured during a visit to the physician's office (8 am to $11 \mathrm{am}$ ) [16]. Briefly, measurements were made in a quiet environment using a mercury sphygmomanometer with the patient in a sitting position after 5 min of rest. BP was not measured if the patient had engaged in recent physical activity, used tobacco, ingested caffeine, or eaten within the previous $30 \mathrm{~min}$. Values of SBP and DBP values (Korotkoff's phase I and phase V, respectively) in each visit were used to obtain a minimum of two BP measurements at intervals of $\geq 1 \mathrm{~min}$. For each visit (baseline and after the drug shift), the reported values of office BP were the mean of values recorded during the 2 days in which the ABP device was installed and removed. For all patients, sphygmomanometric measurements were undertaken by the same physician, who was not aware of the results of ABP recordings.

3. Cardiac assessment: Cardiac structure was assessed by two investigators trained for this purpose before starting the study. Left ventricular volumes, mass, systolic function and diastolic function were assessed using 2-dimensional echocardiography. Left ventricular mass was calculated using the Duvereux method [17]. The left ventricular mass index (LVMI) was obtained by calculating left ventricular mass to height ${ }^{2.7}$ [18]. Left ventricular systolic function was assessed by left ventricular ejection fraction, and diastolic function was assessed by early mitral inflow filling velocity $(E)$, peak mitral filling velocity at atrial contraction (A), E/A deceleration time of the mitral $\mathrm{E}$ wave and tissue Doppler velocity of the mitral annulus [19]. 
4. Carotid ultrasonography: Carotid intima-media thickness (cIMT) was assessed by two trained investigators before study commencement. A SonoSite MicroMaxx Ultrasound System paired with a $5-10-\mathrm{MHz}$ Multifrequency High-resolution linear transducer (Bothell, WA, USA) with Sono-Calc IMT software was used for taking automatic measurements of cIMT. This was achieved by averaging three measurements taken on each carotid artery (anterior, lateral and posterior directions) and measuring the distance between the leading edge of the lumen-intima interface and the leading edge of the collagenous upper layer of the adventitia using high-resolution B-mode ultrasonography. Measurements were taken in areas free of obvious atherosclerotic plaque around the level of the carotid bifurcation.

5. Renal assessment: Kidney damage was assessed by measuring the serum concentrations of creatinine, which is measured by the enzymatic method, traceable to the isotope dilution mass spectrometry. The estimated glomerular filtration rate (eGFR) was calculated using a modified version of the Modification of Diet in Renal Disease (MDRD) equation based on data from Chinese CKD patients as follows [20]:

eGFR $=175 \times$ standardized Scr $r^{-1.234} \times$ age $e^{-0.179} \times 0.79($ if female $)$

6. Other data collection: We also collected urine samples from 07:00 to 07:00 the next day to detect the extent of 24-h proteinuria. These patients were asked to void their bladders at 07:00 to ensure valid results. Proteinuria was measured by immunoturbidimetry. In addition, medical history, including demographic and laboratory data [serum cystatin C, cholesterol, triglycerides (TGs), high-density lipo-protein-cholesterol (HDL-C), low-density lipoprotein-cholesterol (LDL-C), fasting glucose, homocysteine, calcium , phosphate, and intact parathyroid hormone (iPTH)] as well as current therapy were obtained at the initial study visit. The experimental data were measured using a Hitachi 7180 biochemistry auto-analyzer (Tokyo, Japan).

\section{Statistical analyses}

Continuous variables were expressed as the mean \pm standard deviation (SD). Frequency distributions were used for qualitative variables. Non-parametric variables are expressed as median and interquartile range. The log transformation for proteinuria was done in view of the skewed distribution of these data, and Ln (proteinuria +1 ) was used because some patients had low levels of proteinuria.
One-way analysis (ANOVA) or non-parametric test was used to analyze differences among the quartiles of AASI distribution. Pearson's correlation coefficient was employed to estimate the relationship between quantitative variables, and the chi-squared test was used to associate qualitative variables. We employed multivariate linear regression models to study the association of indices of renal function (levels of cystatin $C$, serum creatinine, eGFR and proteinuria) and cardiovascular damage (LVMI, E/A ratio, cIMT) with AASI values. All values are two-tailed. $p<0.05$ was considered significant. Data were analyzed using SPSS ver18.0 (SPSS, Chicago, IL, USA).

\section{Results}

1 Clinical characteristics of patients Of 583 CKD patients (mean age, $43 \pm 16$ years; male/female ratio, 1.45$)$ only $49(8.4 \%)$ had diabetes mellitus (DM) but 109 (18.7\%) had IgA nephropathy. The mean AASI was $0.54 \pm 0.17$. Univariate analyses showed that AASI was positively related with age $(r=0.263, P<0.001)$. The AASI in patients with IgA nephropathy was $0.47 \pm 0.18$, which was lower than non-IgA nephropathy patients $(P<0.05)$. The AASI in DM patients was $0.57 \pm 0.11$, which was similar to that seen in non-DM patients. The main clinical characteristics of the study population across the quartiles of AASI distribution are shown in Table 1. Patients with a higher AASI were older, and had: higher SBP (in the clinic, over 24-h, at daytime and at bedtime); higher mean BP; smaller variability in DBP (mean, at daytime and at bedtime); lower eGFR; higher serum levels of cystatin C; lower levels of hemoglobin; higher serum levels of phosphate; iPTH and calcium $\times$ phosphate; higher LVMI and CIMT and a higher prevalence of carotid arterial plaques.

2 AASI is closely correlated with clinical parameters Univariate analyses showed that AASI was positively correlated with: serum levels of phosphate $(r=0.178, P<0.001)$; iPTH $(r=0.179, P<0.001)$ and calcium $\times$ phosphate $(r=0.163, P<0.001)$; serum levels of uric acid $(r=0.107, P=0.01)$, and negatively correlated with levels of hemoglobin $(r=-0.203$, $P<0.001)$ and HDL-C $(r=-0.096, P=0.025)$ (Table 2). Univariate analyses showed that AASI was positively correlated with SBP in the clinic $(r=0.168$, $P<0.001)$, over $24 \mathrm{~h}(r=0.311, P<0.001)$, while at daytime $(r=0.322, P<0.001)$ and bedtime $(r=0.196$, $P<0.001)$. It was also positively correlated with mean BP $(r=0.226, P<0.001)$ and heart rate $(r=0.09, P=0.017)$. AASI was negatively correlated with the variability of DBP over $24 \mathrm{~h}(r=-0.188$, 
Table 1 Characteristics of the study population by quartiles of AASI distribution

\begin{tabular}{|c|c|c|c|c|}
\hline Variables & $\begin{array}{l}\text { Quartile1 } \\
(<0.42) \\
(n=145)\end{array}$ & $\begin{array}{c}\text { Quartile2 } \\
(0.42 \sim 0.53) \\
(n=146)\end{array}$ & $\begin{array}{c}\text { Quartile3 } \\
(0.54 \sim 0.63) \\
(n=146)\end{array}$ & $\begin{array}{l}\text { Quartile4 } \\
(>0.63) \\
(n=146)\end{array}$ \\
\hline Age (years) & $36 \pm 14$ & $43 \pm 16^{*}$ & $44 \pm 17^{*} \#$ & $48 \pm 17^{*} \# \&$ \\
\hline Male:female ratio & 81: 59 & $87: 59$ & $87: 60$ & $90: 60$ \\
\hline Number of diabetic & 6 & 11 & 15 & 17 \\
\hline ACEls or ARBs (\%) & 50.4 & 55.2 & 58.1 & 63.3 \\
\hline$\overline{\mathrm{CCB}(\%)}$ & 18.4 & 21.5 & 24.6 & 27.8 \\
\hline Proteinuria (g/24h) & $1.2(0.4-4.1)$ & $1.3(0.5-3.3)$ & $1.4(0.5-3.9)$ & $1.2(0.6-3.2)$ \\
\hline Hemoglobin $(\mathrm{g} / \mathrm{L})$ & $121 \pm 29$ & $114 \pm 30$ & $113 \pm 30^{*}$ & $105 \pm 31^{*} \# \&$ \\
\hline Albumin (g/L) & $33 \pm 9$ & $35 \pm 22$ & $33 \pm 9$ & $35 \pm 8$ \\
\hline Globulin (g/L) & $23 \pm 5$ & $24 \pm 5$ & $24 \pm 6$ & $25 \pm 6$ \\
\hline Serum creatinine $(\mu \mathrm{mol} / \mathrm{L})$ & $235 \pm 215$ & $329 \pm 216^{*}$ & $338 \pm 261^{*} \#$ & $445 \pm 254^{*} \# \&$ \\
\hline Serum Cystatin C (mg/L) & $1.9 \pm 1.5$ & $2.4 \pm 1.8^{*}$ & $2.6 \pm 2.0^{*} \#$ & $3.5 \pm 2.5^{*} \# \&$ \\
\hline eGFR-MDRD (ml/min/1.73m²) & $81(22-115)$ & $51(10-103)^{*}$ & $47(12-102)^{*}$ & $27(6-89)^{*} \# \&$ \\
\hline Cholesterol (mmol/L) & $6.3 \pm 3.1$ & $6.2 \pm 3.0$ & $6.2 \pm 3.5$ & $5.5 \pm 2.8$ \\
\hline Triglyceride $(\mathrm{mmol} / \mathrm{L})$ & $2.1 \pm 1.5$ & $2.2 \pm 1.4$ & $2.5 \pm 2.4$ & $2.0 \pm 1.9$ \\
\hline $\mathrm{LDL}-\mathrm{C}(\mathrm{mmol} / \mathrm{L})$ & $4.1 \pm 2.5$ & $4.0 \pm 2.0$ & $3.9 \pm 2.5$ & $3.5 \pm 2.1$ \\
\hline $\mathrm{HDL}-\mathrm{C}(\mathrm{mmol} / \mathrm{L})$ & $1.3 \pm 0.5$ & $1.2 \pm 0.5$ & $1.2 \pm 0.5$ & $1.2 \pm 0.4$ \\
\hline Glucose (mmol/L) & $5.0 \pm 1.8$ & $5.1 \pm 1.9$ & $5.2 \pm 1.8$ & $5.3 \pm 2.0$ \\
\hline Calcium (mg/dL) & $8.6 \pm 0.8$ & $8.7 \pm 0.9$ & $8.4 \pm 1.0 \#$ & $8.5 \pm 0.9$ \\
\hline Phosphate (mmol/L) & $4.1 \pm 1.1$ & $4.5 \pm 1.6^{*}$ & $4.5 \pm 1.6^{*}$ & $4.9 \pm 1.8^{*} \# \&$ \\
\hline Calcium $\times$ Phosphate $\left(\mathrm{mg}^{2} / \mathrm{dl}^{2}\right)$ & $35 \pm 8$ & $39 \pm 13^{*}$ & $37 \pm 10$ & $41 \pm 13^{*} \&$ \\
\hline iPTH (pg/ml) & $116 \pm 98$ & $139 \pm 94$ & $155 \pm 130^{*}$ & $217 \pm 167^{*} \# \&$ \\
\hline Urine acid (mmol/L) & $433 \pm 150$ & $460 \pm 156$ & $466 \pm 146$ & $470 \pm 157$ \\
\hline homocysteine $(\mu \mathrm{mol} / \mathrm{L})$ & $14.3 \pm 8.6$ & $18.2 \pm 10.0^{*}$ & $16.8 \pm 8.4^{*}$ & $16.2 \pm 7.9^{*}$ \\
\hline LVEF (\%) & $63 \pm 18$ & $64 \pm 16$ & $66 \pm 12$ & $65 \pm 14$ \\
\hline LVMI $\left(\mathrm{g} / \mathrm{m}^{2.7}\right)$ & $43 \pm 15$ & $49 \pm 17^{*}$ & $56 \pm 27^{*}$ & $54 \pm 19^{*}$ \\
\hline E/A ratio & $1.3 \pm 0.4$ & $1.1 \pm 0.4^{*}$ & $1.2 \pm 0.6$ & $1.1 \pm 0.4^{*}$ \\
\hline $\mathrm{cIMI}(\mathrm{mm})$ & $0.61 \pm 0.22$ & $0.64 \pm 0.21$ & $0.68 \pm 0.25$ & $0.73 \pm 0.35^{*} \#$ \\
\hline Number of plaque & 6 & 10 & $19^{*}$ & $20^{*}$ \\
\hline Clinic- SBP (mmHg) & $135 \pm 21$ & $141 \pm 23^{*}$ & $146 \pm 24^{*}$ & $145 \pm 26^{*}$ \\
\hline Clinic-DBP (mmHg) & $84 \pm 14$ & $85 \pm 15$ & $87 \pm 14$ & $84 \pm 14$ \\
\hline average-SBP (mmHg) & $126 \pm 17$ & $131 \pm 20^{*}$ & $138 \pm 20^{*} \#$ & $142 \pm 20^{*} \#$ \\
\hline average-DBP (mmHg) & $78 \pm 11$ & $79 \pm 12$ & $82 \pm 12$ & $81 \pm 11$ \\
\hline SBP-daytime $(\mathrm{mmHg})$ & $128 \pm 17$ & $134 \pm 18^{*}$ & $138 \pm 19^{*} \#$ & $143 \pm 19^{*} \# \&$ \\
\hline DBP-daytime $(\mathrm{mmHg})$ & $79 \pm 11$ & $81 \pm 11$ & $82 \pm 12$ & $82 \pm 11$ \\
\hline SBP-bedtime $(\mathrm{mmHg})$ & $110 \pm 31$ & $122 \pm 39^{*}$ & $128 \pm 31^{*}$ & $135 \pm 28^{*} \# \&$ \\
\hline DBP-bedtime $(\mathrm{mmHg})$ & $67 \pm 19$ & $74 \pm 16^{*}$ & $77 \pm 20^{*}$ & $78 \pm 15^{*} \#$ \\
\hline Decline of SBP-bedtime & $7.9 \pm 7.0$ & $6.3 \pm 6.0$ & $6.0 \pm 14$ & $4.5 \pm 11.4$ \\
\hline Decline of DBP-bedtime & $10.6 \pm 8.5^{*}$ & $7.2 \pm 8.9^{*}$ & $6.6 \pm 13.6^{*}$ & $3.6 \pm 11.5^{*} \&$ \\
\hline 24h-MBP & $93 \pm 12$ & $96 \pm 14$ & $100 \pm 13$ & $101 \pm 113^{*} \#$ \\
\hline Variability of SBP (\%) & $14 \pm 8$ & $15 \pm 8$ & $15 \pm 11$ & $13 \pm 8$ \\
\hline Variability of DBP (\%) & $24 \pm 20$ & $20 \pm 9^{*}$ & $18 \pm 10^{*}$ & $16 \pm 6^{*} \#$ \\
\hline Variability of SBP-daytime (\%) & $13 \pm 5$ & $14 \pm 5$ & $13 \pm 4 \#$ & $12 \pm 4 \#$ \\
\hline
\end{tabular}


Table 1 Characteristics of the study population by quartiles of AASI distribution (Continued)

\begin{tabular}{lcccc}
\hline Variability of DBP -daytime (\%) & $21 \pm 7$ & $19 \pm 7^{*}$ & $17 \pm 5^{*} \#$ & $16 \pm 5^{*} \# \&$ \\
\hline Variability of SBP-bedtime (\%) & $12 \pm 6$ & $12 \pm 5$ & $11 \pm 6$ & $10 \pm 5^{*} \#$ \\
\hline Variability of DBP -bedtime (\%) & $16 \pm 7$ & $16 \pm 7$ & $14 \pm 6^{*} \#$ & $13 \pm 5^{*} \#$ \\
\hline Pulse (beat/min) & $75 \pm 10$ & $78 \pm 10^{*}$ & $78 \pm 11$ & $78 \pm 11$ \\
\hline AASI (IU) & $0.32 \pm 0.08$ & $0.48 \pm 0.03^{*}$ & $0.59 \pm 0.03^{*} \#$ & $0.74 \pm 0.08^{*} \# \&$ \\
\hline
\end{tabular}

(AASI: ambulatory arterial stiffness index, ARB: angiotensin receptor blocker, ACEl: angiotensin-converting enzyme inhibitors, BMI: body mass index, CCB: calcium channel blocker, eGFR: estimated glomerular filtration rate; LDL-C: low-density lipoprotein cholesterol; HDL-C: high-density lipoprotein cholesterol; iPTH: intact parathyroid hormone; LVEF: Left ventricular ejection fraction; LVMI: Left ventricular mass index; E: early mitral inflow filling velocity; A: peak mitral filling velocity at atrial contraction; cIMl: Carotid intima-media thickness. Serum calcium is corrected by the following formula: [calcium] $(\mathrm{mg} / \mathrm{dL})=$ measured [calcium] $+(4.0-[\mathrm{serum}$ albumin $(\mathrm{mg} / \mathrm{dl})]) \times 0.8$; SBP: systolic blood pressure; DBP: diastolic blood pressure; ABPM: ambulatory blood pressure monitoring. *indicated contro with Quartile1 P<0.05, \# indicated contro with Quartile2 $\mathrm{P}<0.05$, \&\# indicated contro with Quartile3 $\mathrm{P}<0.05)$.

$P<0.001)$, while at daytime $(r=-0.283, P<0.001)$ and bedtime $(r=-0.162, P<0.001)$. AASI was also negatively correlated with SBP while at daytime $(r=-0.114, P=0.006)$ and bedtime $(r=-0.091$, $P=0.029)$ as well as the decline in bedtime SBP $(r=-0.124, P=0.003)$ and DBP $(r=-0.226$, $P<0.001$ ) (Table 2).

3 AASI is closely correlated with renal function Univariate analyses revealed AASI was negatively correlated with the eGFR $(r=-0.200, P<0.001)$ and positively correlated with serum levels of cystatin $C$ $(r=0.296, P<0.001)$ and serum creatinine $(r=0.182$, $P<0.001)$ Multiple linear regression analyses were done to evaluate the relationship between eGFR, serum levels of cystatin $C$ and creatinine with AASI. The eGFR was independently correlated with age ( $\beta=-1.477), 24-\mathrm{h}$ mean BP $(\beta=-1.538)$, the decline ratio of bedtime SBP $(\beta=0.534)$ and the AASI $(\beta=-20.033)$. Serum levels of cystatin $C$ were independently correlated with age $(\beta=0.022)$, 24-h mean BP $(\beta=0.045)$, the decline in the ratio of bedtime SBP $(\beta=-0.038)$, and AASI $(\beta=1.795)$. Serum creatinine were independently correlated with sex ( $\beta=-91.657)$, 24-h mean BP $(\beta=9.259)$, the decline in the ratio of bedtime SBP $(\beta=-8.568)$ and AASI $(\beta=262.347)$. A significant relationship between AASI and proteinuria was not found when univariate and multiple linear regression analyses were undertaken (Tables 2 and 3).

4 AASI is closely correlated with the LVMI Univariate analyses showed that AASI was positively correlated with the LVMI $(r=0.205$, $P<0.001)$ and cIMT $(r=0.156, P=0.002)$, and was negatively related to the E/A ratio $(r=-0.114$, $P=0.018)$. However, we found that the LVMI (but not the cIMT and E/A ratio) was independently correlated with AASI $(\beta=1.712)$ after multiple linear regression analyses were carried out (Table 4).

\section{Discussion}

In this clinical trial, we initially explored the relationship between AASI and target-organ damage in CKD patients in China. The mean AASI in our patients was 0.54, which was higher than hypertensive patients in China [21]. Patients with a higher AASI were older and had: worse renal function; more chronic CKD (anemia, higher serum levels of phosphate and parathyroid hormone); higher LVMI and CIMT; and higher BP. AASI was correlated with renal function (eGFR and serum levels of cystatin C) and LVMI according to univariate and multivariate analyses. This finding suggested that the AASI was a potential index for the assessment of cardiovascular and renal damage. Furthermore, AASI was significantly correlated with variability in $\mathrm{BP}$ pressure, which suggested that AASI might be a parameter for assessing $\mathrm{BP}$ variability.

AASI is derived from BP parameters and thus the multiple reports on the correlation between AASI and pulse pressure or systolic BP do not necessarily reflect a true biological relationship. In a recent study using a computer model of the arterial circulation, arterial stiffness along with vascular resistance and heart rate were identified as the main determinants of AASI [22]. Moreover, several studies have shown night BP reduction to have considerable impact on AASI values [23]. Thus, AASI cannot be considered as a marker of arterial stiffness but rather as a composite index reflecting cardiovascular properties, BP variability and diurnal cycle. Our results showed AASI was correlated with target organ damage and $\mathrm{BP}$ variability in CKD patients. This is the first report on the relationship between AASI and target-organ damage in a large cohort of Chinese CKD patients based on cross-sectional data.

IgA nephropathy is the most common cause of primary glomerulonephritis in "developing" countries such as China [24], whereas diabetic nephropathy is the main cause of end stage of renal disease in developed countries [25]. Almost 20\% patients in our study had IgA nephropathy, and less than $10 \%$ patients suffered from diabetic nephropathy, which is completely different from that seen in western countries. Patients with IgA 
Table 2 Baseline characteristics of clinical data and correlation coefficient with AASI

\begin{tabular}{|c|c|c|c|}
\hline Variables & $N=583$ & Correlation coefficient with AASI & $\mathrm{P}$ value \\
\hline Age (years) & $43 \pm 16$ & 0.263 & $<0.001$ \\
\hline Duration (months) & $24 \pm 17$ & 0.075 & 0.074 \\
\hline $\mathrm{BMI}\left(\mathrm{kg} / \mathrm{m}^{2}\right)$ & $23 \pm 4$ & -0.019 & 0.652 \\
\hline Ln (Proteinuria +1$)$ & $1.0 \pm 0.7$ & -0.007 & 0.867 \\
\hline Hemoglobin (g/L) & $113 \pm 30$ & -0.203 & $<0.001$ \\
\hline Albumin $(\mathrm{g} / \mathrm{L})$ & $34 \pm 13$ & 0.004 & 0.931 \\
\hline Globulin (g/L) & $24 \pm 6$ & 0.098 & 0.02 \\
\hline Serum Cystatin C (mg/L) & $2.6 \pm 2.1$ & 0.296 & $<0.001$ \\
\hline Serum creatinine $(\mu \mathrm{mol} / \mathrm{L})$ & $339 \pm 222$ & 0.182 & $<0.001$ \\
\hline eGFR-MDRD (ml/min/1.73m²) & $59 \pm 48$ & -0.200 & $<0.001$ \\
\hline Cholesterol (mmol/L) & $6.1 \pm 3.1$ & -0.089 & 0.039 \\
\hline Triglyceride $(\mathrm{mmol} / \mathrm{L})$ & $2.2 \pm 1.8$ & -0.028 & 0.517 \\
\hline $\mathrm{LDL}-\mathrm{C}(\mathrm{mmol} / \mathrm{L})$ & $3.9 \pm 2.4$ & -0.08 & 0.062 \\
\hline $\mathrm{HDL}-\mathrm{C}(\mathrm{mmol} / \mathrm{L})$ & $1.2 \pm 0.5$ & -0.096 & 0.025 \\
\hline Glucose $(\mathrm{mmol} / \mathrm{L})$ & $5.2 \pm 1.5$ & 0.079 & 0.057 \\
\hline Calcium (mg/dL) & $8.6 \pm 0.8$ & -0.079 & 0.056 \\
\hline Phosphate (mmol/L) & $4.5 \pm 1.6$ & 0.178 & $<0.001$ \\
\hline Calcium $\times$ Phosphate $\left(\mathrm{mg}^{2} / \mathrm{dl}^{2}\right)$ & $36 \pm 10$ & 0.163 & $<0.001$ \\
\hline $\mathrm{iPTH}(\mathrm{pg} / \mathrm{ml})$ & $157 \pm 73$ & 0.179 & $<0.001$ \\
\hline Uric acid (mmol/L) & $458 \pm 163$ & 0.107 & 0.010 \\
\hline homocysteine $(\mu \mathrm{mol} / \mathrm{L})$ & $16.4 \pm 8.8$ & 0.067 & 0.143 \\
\hline LVEF (\%) & $63 \pm 16$ & 0.036 & 0.445 \\
\hline LVMI $\left(\mathrm{g} / \mathrm{m}^{2.7}\right)$ & $51 \pm 21$ & 0.205 & $<0.001$ \\
\hline E/A ratio & $1.2 \pm 0.5$ & -0.114 & 0.018 \\
\hline $\mathrm{clMl}(\mathrm{mm})$ & $0.7 \pm 0.3$ & 0.156 & 0.002 \\
\hline Clinic-SBP (mmHg) & $142 \pm 24$ & 0.168 & $<0.001$ \\
\hline Clinic-DBP (mmHg) & $85 \pm 14$ & -0.006 & 0.881 \\
\hline average-SBP (mmHg) & $134 \pm 20$ & 0.311 & $<0.001$ \\
\hline average-DBP $(\mathrm{mmHg})$ & $80 \pm 12$ & 0.128 & 0.002 \\
\hline SBP-daytime (mmHg) & $136 \pm 19$ & 0.322 & $<0.001$ \\
\hline DBP-daytime $(\mathrm{mmHg})$ & $81 \pm 11$ & 0.166 & 0.005 \\
\hline SBP-bedtime $(\mathrm{mmHg})$ & $124 \pm 31$ & 0.296 & $<0.001$ \\
\hline DBP-bedtime $(\mathrm{mmHg})$ & $74 \pm 18$ & 0.216 & $<0.001$ \\
\hline Decline of SBP-bedtime & $6 \pm 11$ & -0.124 & 0.003 \\
\hline Decline of DBP-bedtime & $7 \pm 11$ & -0.226 & $<0.001$ \\
\hline 24h-MBP & $98 \pm 13$ & 0.226 & $<0.001$ \\
\hline Variability of SBP (\%) & $14 \pm 9$ & -0.039 & 0.352 \\
\hline Variability of DBP (\%) & $19 \pm 13$ & -0.188 & $<0.001$ \\
\hline Variability of SBP-daytime (\%) & $13 \pm 4$ & -0.114 & 0.006 \\
\hline Variability of DBP-daytime (\%) & $18 \pm 6$ & -0.283 & $<0.001$ \\
\hline Variability of SBP-bedtime (\%) & $11 \pm 6$ & -0.091 & 0.029 \\
\hline Variability of DBP-bedtime (\%) & $15 \pm 6$ & -0.162 & $<0.001$ \\
\hline Pulse (beat/min) & $77 \pm 10$ & 0.09 & 0.017 \\
\hline AASI (IU) & $0.54 \pm 0.17$ & 1.000 & $<0.001$ \\
\hline
\end{tabular}

(AASI: ambulatory arterial stiffness index, BMI: body mass index, eGFR: estimated glomerular filtration rate; LDL-C: low-density lipoprotein cholesterol; HDL-C: high-density lipoprotein cholesterol; iPTH: intact parathyroid hormone; LVEF: Left ventricular ejection fraction; LVMI: Left ventricular mass index; E: early mitral inflow filling velocity; A: peak mitral filling velocity at atrial contraction; cIMl: Carotid intima-media thickness. Serum calcium is corrected by the following formula: [calcium] $(\mathrm{mg} / \mathrm{dL})=\mathrm{measured}$ [calcium] + ( 4.0-[serum albumin (mg/dl)]) × 0.8; SBP: systolic blood pressure; DBP: diastolic blood pressure; ABPM: ambulatory blood pressure monitoring). 
Table 3 Multiple linear regression analysis: relationship between renal parameters (eGFR, Serum Cystatin C, serum creatinine and proteinuira) and AASI in CKD patients

\begin{tabular}{|c|c|c|c|}
\hline Variables & Not standardized b & Confidence interval $95 \%$ & $P$ value \\
\hline \multicolumn{4}{|c|}{ Dependent variable: eGFR by MDRD formula (Adjusted $R^{2}=0.322$ ) } \\
\hline Age (year) & -1.477 & $-1.685 \sim-1.263$ & $<0.001$ \\
\hline 24h-MBP & -1.538 & $-1.770 \sim-1.291$ & $<0.001$ \\
\hline decline ratio of bedtime SBP & 0.534 & $0.225 \sim-0.834$ & 0.001 \\
\hline AASI (IU) & -20.033 & $-40.263 \sim-0.807$ & 0.037 \\
\hline \multicolumn{4}{|c|}{ Dependent variable: Serum Cystatin C (Adjusted $R^{2}=0.304$ ) } \\
\hline Age (year) & 0.022 & $0.013 \sim 0.032$ & $<0.001$ \\
\hline 24h-MBP & 0.045 & $0.034 \sim 0.057$ & $<0.001$ \\
\hline Decline ratio of bedtime SBP & -0.038 & $-0.054 \sim-0.021$ & $<0.001$ \\
\hline AASI (IU) & 1.795 & $0.883 \sim 2.707$ & $<0.001$ \\
\hline \multicolumn{4}{|c|}{ Dependent variable: Serum creatinine (Adjusted $R^{2}=0.213$ ) } \\
\hline Sex & -91.657 & $-153.032 \sim-30.283$ & 0.003 \\
\hline $24 h-M B P$ & 9.259 & $6.940 \sim 11.579$ & $<0.001$ \\
\hline Decline ratio of bedtime SBP & -8.568 & $-11.744 \sim-5.392$ & $<0.001$ \\
\hline AASI (IU) & 262.347 & $78.405 \sim 446.289$ & 0.005 \\
\hline \multicolumn{4}{|c|}{ Dependent variable: $\operatorname{In}($ proteinuria +1$)\left(\right.$ Adjusted $\left.R^{2}=0.034\right)$} \\
\hline Age & -0.008 & $-0.012 \sim-0.004$ & $<0.001$ \\
\hline 24h-MBP & 0.009 & $0.004 \sim 0.014$ & $<0.001$ \\
\hline
\end{tabular}

Adjusted Variables: Age; Sex: (male $=1$; female $=2)$.

Independent variable: In (24Pro + 1), 24h-MBP, decline ratio of bedtime SBP, decline ratio of bedtime DBP, AASI (IU).

(AASI: ambulatory arterial stiffness index, eGFR: estimated glomerular filtration rate, MBP: mean blood pressure; SBP: systolic blood pressure).

nephropathy are younger, and show less vascular complications, whereas patients with diabetic nephropathy are older and have more micro and macro vascular complications. All these factors mentioned in Chinese CKD patients might lead to different roles of AASI in patients from western countries. We found that AASI in IgA patients was lower than non-IgA nephropathy patients, which was related to being younger and having slight renal damage. However the number of subjects with diabetic nephropathy in the present study was small, we need to study more diabetic patients to confirm the role of AASI in patients with diabetic nephropathy.

CVD is a leading cause of morbidity and mortality, accounting for $30-40 \%$ of deaths in patients with renal failure. Left ventricular hypertrophy, left ventricular dilatation, systolic dysfunction and diastolic dysfunction

Table 4 Multiple linear regression analysis: relationship between cardiovascular damage parameter (Left ventricular mass index, E/A ratio, Carotid intima-media thickness and Carotid aterial plaque) and AASI in CKD patients

\begin{tabular}{|c|c|c|c|}
\hline Variables & Not standardized $\beta$ & Confidence interval $95 \%$ & $\mathrm{P}$ value \\
\hline \multicolumn{4}{|c|}{ Dependent variable: Left ventricular mass index (Adjusted $R^{2}=0.329$ ) } \\
\hline eGFR-MDRD (ml/min/1.73m²) & -0.143 & $-0.183 \sim-0.103$ & $<0.001$ \\
\hline $\mathrm{BMI}$ & 1.392 & $1.020 \sim 1.764$ & $<0.001$ \\
\hline $24 \mathrm{~h}-\mathrm{MBP}$ & 0.163 & $0.012 \sim 0.313$ & 0.034 \\
\hline decline ratio of bedtime SBP & -0.274 & $-0.451 \sim-0.096$ & 0.003 \\
\hline AASI (IU) & 1.712 & $0.191 \sim 3.233$ & 0.027 \\
\hline \multicolumn{4}{|c|}{ Dependent variable: $E / A$ ratio (Adjusted $R^{2}=0.283$ ) } \\
\hline Age & -0.014 & $-0.016 \sim-0.011$ & $<0.001$ \\
\hline 24h-MBP & -0.006 & $-0.009 \sim-0.003$ & $<0.001$ \\
\hline \multicolumn{4}{|c|}{ Dependent variable: Carotid intima-media thickness (Adjusted $R^{2}=0.305$ ) } \\
\hline Age & 0.009 & $0.008 \sim 0.010$ & $<0.001$ \\
\hline Sex & -0.070 & $-0.115 \sim-0.024$ & 0.003 \\
\hline
\end{tabular}

Adjusted Variables: Age; Sex: (male = 1; female $=2$ ).

Independent variable: eGFR-MDRD ( $\left.\mathrm{ml} / \mathrm{min} / 1.73 \mathrm{~m}^{2}\right), \mathrm{BMI}, 24 \mathrm{~h}-\mathrm{MBP}$, decline ratio of bedtime SBP, decline ratio of bedtime DBP, AASI (IU).

(AASI: ambulatory arterial stiffness index, eGFR: estimated glomerular filtration rate, MBP: mean blood pressure; SBP: systolic blood pressure). 
are very common and independently associated with mortality in CKD patients [26]. The cIMT is a marker for the presence and severity of arteriosclerosis, and has been associated with risk factors for all CVDs, all-cause mortality, and cardiovascular mortality [27]. AASI is a well-known predictor of cardiovascular mortality in hypertensive patients [28]. However, there are few reports on the relationship between AASI and cardiovascular damages. We are the first to study this relationship in Chinese CKD patients. We found that patients with higher AASI values had a higher LVMI and cIMT as well as a higher prevalence of carotid arterial plaques. Also, AASI was positively related to the LVMI and cIMT. Our results indicated that AASI was closely related to the traditional risk factors of CVD such as LVMI and cIMT. AASI might be a potential marker for the assessment of cardiovascular damage in CKD patients and more clinical trials are needed to confirm this finding.

Proteinuria is a strong, independent predictor of ESRD in a mass-screening setting. Even a slight increase in proteinuria is an independent risk factor for ESRD [29]. Proteinuria is strongly associated with arterial stiffness and endothelial dysfunction [30], so proteinuria could reflect arterial stiffness-associated increases. Data from essential hypertension showed arterial stiffness correlated with albumin excretion rate [31]. However, some authors have reported that the 24-h albumin excretion rate did not correlate with AASI [32], and baseline arterial stiffness did not correlate with the incidence of microalbuminuria [33]. A significant relationship between proteinuria and the AASI was not found in our study even we used different statistical methods, which might be related with the complexcity of proteinuria in CKD patients. However, we found AASI correlate with renal function (assessed by eGFR, serum cystanic $C$ and creatinine), which suggested AASI might be a parameter for the assessment for the progression of CKD, and more clinical trials are needed to confirm this finding.

Our study had several strengths. We are the first to report the close relationship between AASI and cardiovascular/renal damage in CKD patients. Also, the sample size was large. Finally, we reported the relationship between AASI and renal cardiovascular damage in Chinese CKD patients. However, we need larger perspective studies to confirm the role of AASI in Chinese CKD patients.

\section{Conclusions}

The present study provided evidence that AASI is associated with renal function and cardiovascular risk factors in Chinese CKD patients. AASI might be a potential index for assessment in CKD patients. More clinical studies with larger cohorts of patients are needed to confirm the role of AASI in Chinese CKD patients.

\section{Abbreviations}

AASI: Ambulatory arterial stiffness index; ARB: Angiotensin receptor blocker; ACEl: Angiotensin-converting enzyme inhibitors; BMI: Body mass index; CCB: Calcium channel blocker; cIMl: Carotid intima-media thickness; eGFR: Estimated glomerular filtration rate; LDL-C: Low-density lipoprotein cholesterol; HDL-C: High-density lipoprotein cholesterol; iPTH: Intact parathyroid hormone; LVEF: Left ventricular ejection fraction; LVMI: Left ventricular mass index; E: Early mitral inflow filling velocity; A: Peak mitral filling velocity at atrial contraction.

\section{Competing interests}

The authors declare that they have no competing interests.

\section{Authors' contributions}

Conceived and designed the experiments: CW, JZ and TL. Performed the experiments: XL, CL, WG and ZY. Analyzed the data: JZ and CW. Contributed reagents/materials/analysis tools: HP and ZY. Wrote the paper: CW. Review the manuscript for intellectual content: Dr CW, JZ and TL. All authors read and approved the final manuscript.

\section{Acknowledgements}

We would like to thank all patients and their families for participating in this study.

Received: 4 December 2012 Accepted: 7 November 2013

Published: 19 November 2013

\section{References}

1. Couser WG, Remuzzi G, Mendis S, Tonelli M: The contribution of chronic kidney disease to the global burden of major noncommunicable diseases. Kidney Int 2011, 80:1258-1270.

2. Siasos G, Tousoulis D, Michalea S, Oikonomou E, Kolia C, Kioufis S, Synetos A, Vlasis K, Papavassiliou AG, Stefanadis C: Biomarkers determining cardiovascular risk in patients with kidney disease. Curr Med Chem 2012, 19(16):2555-2571.

3. Gosse P, Safar ME: Arterial stiffness and plasma creatinine in untreated hypertensive patients. Am J Hypertension 2005, 18:1140-1145.

4. Chue CD, Townend JN, Steeds RP, Ferro CJ: Republished paper: arterial stiffness in chronic kidney disease: causes and consequences. Postgrad Med J 2010, 86:560-566.

5. Hansen TW, Staessen JA, Torp-Pedersen C, Rasmussen S, Li Y, Dolan E, Thijs L, Wang JG, O'Brien E, Ibsen H, Jeppesen J: Ambulatory arterial stiffness index predicts stroke in a general population. J Hypertens 2006, 24:2247-2253.

6. Li Y, Wang JG, Dolan E, Gao PJ, Guo HF, Nawrot T, Stanton AV, Zhu DL, O'Brien E, Staessen JA: Ambulatory arterial stiffness index derived from 24-hour ambulatory blood pressure monitoring. Hypertension 2006, 47:359-364.

7. Dolan E, Thijs L, Li Y, Atkins N, McCormack P, McClony S, O'Brien E, Staessen JA, Stanton AV: Ambulatory arterial stiffness index as a predictor of cardiovascular mortality in the Dublin outcome study. Hypertension 2006, 47:365-370.

8. Kikuya M, Staessen JA, Ohkubo T, Thijs L, Metoki H, Asayama K, Obara T, Inoue R, Li Y, Dolan E, Hoshi H, Hashimoto J, Totsune K, Satoh H, Wang JG, O'Brien E, Imai Y: Ambulatory arterial stiffness index and 24-hour ambulatory pulse pressure as predictors of mortality in Ohasama, Japan. Stroke 2007, 38:1161-1166.

9. Muxfeldt ES, Cardoso CR, Dias VB, Nascimento AC, Salles GF: Prognostic impact of the ambulatory arterial stiffness index in resistant hypertension. J Hypertens 2010, 28:1547-1553.

10. Li Y, Hansen TW, Staessen JA: Ambulatory arterial stiffness index: reproducibility of different definitions. Am J Hypertens 2010, 23:129-134.

11. Mulè G, Cottone $S$, Cusimano P, Incalcaterra F, Giandalia M, Costanzo M, Nardi E, Palermo A, Geraci C, Costa R, Cerasola G: Inverse relationship between ambulatory arterial stiffness index and glomerular filtration rate in arterial hypertension. Am J Hypertens 2008, 21:35-40.

12. Leoncini G, Ratto E, Viazzi F, Vaccaro V, Parodi A, Falqui V, Conti N, Tomolillo C, Deferrari G, Pontremoli R: Increased ambulatory arterial stiffness index is associated with target organ damage in primary hypertension. Hypertension 2006, 48:397-403.

13. Gómez-Marcos MA, Recio-Rodríquez Jl, Patino-Alonso MC, Gómez-Sánchez L, Agudo-Conde C, Gómez-Sánchez M, Rodríguez-Sánchez E, García-Ortiz L, Vasorisk Group: Ambulatory arterialstiffness indices and target organ damage in hypertension. BMC Cardiovasc Disord 2012, 27:12. 1. 
14. Stergiou GS, Kollias A, Giovas PP, Papagiannis J, Roussias LG: Ambulatory arterial stiffness index, pulse pressure and pulse wave velocity in children and adolescents. Hypertens Res 2010, 33:1272-1277.

15. Pogue V, Rahman M, Lipkowitz M, Toto R, Miller E, Faulkner M, Rostand S, Hiremath L, Sika M, Kendrick C, Hu B, Greene T, Appel L, Phillips RA, African American Study of Kidney Disease and Hypertension Collaborative Research Group: Disparate estimates of hypertension control from ambulatory and clinic blood pressure measurements in hypertensive kidney disease. Hypertension 2009, 53:20-27.

16. Williams JS, Brown SM, Conlin PR: Videos in clinical medicine: bloodpressure measurement. N Engl J Med 2009, 360:e6.

17. Schiller NB, Shah PM, Crawford M, DeMaria A, Devereux R, Feigenbaum H, Gutgesell H, Reichek N, Sahn D, Schnittger I, et al: Recommendations for quantification of the left ventricle by two-dimensional echocardiography: American society of echocardiography committee on standards, subcommittee on quantitation of two-dimensional echocardiograms. J Am Soc Echocardiography 1989, 2:358-367.

18. Zoccali C, Benedetto FA, Mallamaci F, Tripepi G, Giacone G, Cataliotti A, Seminara G, Stancanelli B, Malatino LS, CREED Investigators: Prognostic impact of the indexation of left ventricular mass in patients undergoing dialysis. J Am Soc Nephrol 2001, 12:2768-2774

19. Ommen S, Nishimura R: A clinical approach to the assessment of left ventricular diastolic function by Doppler echocardiography: update, 2003. Heart 2003, 89:18-23.

20. Ma YC, Zuo L, Chen JH, Luo Q, Yu XQ, Li Y, Xu JS, Huang SM, Wang LN, Huang W, Wang M, Xu GB, Wang HY: Modified glomerular filtration rate estimating equation for Chinese patients with chronic kidney disease. J Am Soc Nephrol 2006, 17:2937-2944.

21. Wang Y, Hu Y, Li Y, Li H, Chu S, Zhu D, Gao P: Association of renal function with the ambulatory arterial stiffness index and pulse pressure in hypertensive patients. Hypertens Res 2012, 35:201-206.

22. Kips JG, Vermeersch SJ, Reymond P, Boutouyrie P, Stergiopulos N, Laurent S, Van Bortel LM, Segers P: Ambulatory arterial stiffness index does not accurately assess arterial stiffness. J Hypertens 2012, 30:574-580.

23. Laugesen E, Erlandsen M, Knudsen ST, Hansen KW, Poulsen PL: Ambulatory arterial stiffness index: a composite index reflecting arterial stiffness, blood pressure variability and patients' diurnal cycle. J Hypertens 2011 29:2278-2279.

24. Peres LA, Matsuo T, Delfino VD, Peres CP, Almeida Netto JH, Ann HK, Camargo MT, Rohde NR, Uscocovich VF: Increase in prevalence of diabetes mellitus as a cause of dialytic end-stage renal disease: analysis of 20 years in the west region of Paraná. Arq Bras Endocrinol Metabol 2007, 51:111-115.

25. USRDS annual data report. ; 2012

26. Van Eps $\mathrm{CL}$, Jeffriess L, Haluska B, Hawley CM, Coombes J, Matsumoto A, Jeffries JK, Johnson DW, Campbell SB, Isbel NM, Mudge DW, Marwick T: Cardiac and vascular structure and function parameters do not improve with alternate nightly home hemodialysis: An interventional cohort study. BMC Nephrol 2011, 12:51-65.

27. Zhang L, Zhao F, Yang Y, Qi L, Zhang B, Wang F, Wang S, Liu L, Wang H: Associations between carotid artery intima-media thickness and cardiovascular risk factors in CKD. Am J Kidney Dis 2005, 46:856-862.

28. Laurent S, Cockcroft J, Van Bortel L, Boutouyrie P, Giannattasio C, Hayoz D, Pannier B, Vlachopoulos C, Wilkinson I, Struijker-Boudier H: European network for non-invasive investigation of large arteries: expert consensus document on arterial stiffness: methodological issues and clinical applications. Eur Heart J 2006, 27:2588-2605

29. Kunitoshi I, Yoshiharu I, Chiho I, et al: Proteinuria and the risk of developing end-stage renal disease. Kidney Int 2003, 63:1468-1474.

30. Agarwal R: Antihypertensive agents and arterial stiffness: relevance to reducing cardiovascular risk in the chronic kidney disease patient. Curr Opin Nephrol Hypertens 2007, 16:409-415.

31. Mulè G, Cottone S, Cusimano P, Palermo A, Geraci C, Nardi E, Castiglia A, Costanzo M, Cerasola G: Unfavourable interaction of microalbuminuria and mildly reduced creatinine clearance on aortic stiffness in essential hypertension. Int J Cardiol 2010, 145:372-375.
32. Robles NR, Mena C, Macias R, Garcia de Vinuesa E, Herrera J, Macias JF: Symmetrical ambulatory arterial stiffness index: relationship with microalbuminuria and renal function. Eur J Int Med 2010, 21:118-122.

33. Upadhyay A, Hwang SJ, Mitchell GF, Vasan RS, Vita JA, Stantchev PI, Meigs JB, Larson MG, Levy D, Benjamin EJ: Fox CS: arterial stiffness in mild-to-moderate CKD. J Am Soc Nephrol 2009, 20:2044-2053.

doi:10.1186/1471-2369-14-257

Cite this article as: Wang et al:: The ambulatory arterial stiffness index and target-organ damage in Chinese patients with chronic kidney disease. BMC Nephrology 2013 14:257.

\section{Submit your next manuscript to BioMed Central and take full advantage of:}

- Convenient online submission

- Thorough peer review

- No space constraints or color figure charges

- Immediate publication on acceptance

- Inclusion in PubMed, CAS, Scopus and Google Scholar

- Research which is freely available for redistribution

Submit your manuscript at www.biomedcentral.com/submit
C BioMed Central 